\title{
Die historischen Angaben in Aelius Aristides Panathenaikos auf ihre Quellen untersucht.
}

\author{
Von
}

Dr. EUGEN BEECKE.

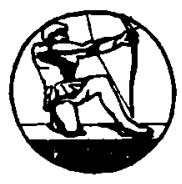

STRASSBURG

VERLAG VON KARL J. TRÜBNER 1908. 
M. DuYont Schauberg, Straßburg i. E. 


\section{BRUNO KEIL}

\section{RICHARD REITZENSTEIN}

ZUGEEIGNETT. 

Die Schriften des Rhetors Aelius Aristides enthalten eine große Anzahl von Angaben aus der griechischen Geschichte von der mythischen Zeit bis zu der Alexanders des Großen; unter diese 'Zeitgrenze geht der Redner ${ }^{1}$ ) entsprechend der Schultradition der griechischen Rhetorik nicht herunter. Die Forschung hat es in erster Linie mit der Bewertung der von diesem bedeutendsten Vertreter der sophistischen Beredsamkeit der Kaiserzeit gebotenen historischen Angaben zu tun; unsere Untersuchung wird zeigen, wie begründet Bauer ${ }^{2}$ ) sagen durfte „unser Urtheil über den Werth der bei ihm erhaltenen Angaben wird sich ebenso zu stellen haben wie bei Ephoros". Ja, in noch weit stärkerem Maße als dieser hat sich Aristides die Glorifizierung und Rhetorisierung jener großen, nach seinem Dafürhalten bisher keineswegs gebührend verherrlichten Ereignisse und Heldentaten Athens zur Aufgabe gestellt. Er ist ein Typus: was diese sophistische Beredsamkeit der Kaiserzeit mit der Überlieferung des Altertums gemacht hat, wird neben den hierüber bereits vorliegenden Untersuchungen und Darstellungen auch die folgende zeigen.

Es ist schon von verschiedenen Seiten der Versuch gemacht worden, für einzelne Schriften unseres Rhetors die historischen Quellen aufzuzeigen. So ist auf die Quellenbe-

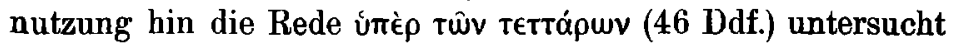
worden, zuerst von Baner, Themistokles, $145 \mathrm{ff}$. dann von Haas ${ }^{3}$ ),

1) In der Romrede, Arist. II, 103, 3 - 107, $21 \mathrm{~K}$., endet der sum-

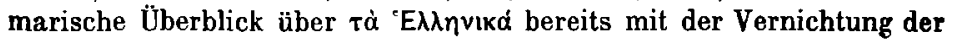
spartanischen Macht bei Leuktra und Thebens Suprematie in Mittelgriechenland (Arist. a. a. 0. 105, 12 ff.).

2) Bauer, Themistokles, Studien und Beiträge zur griechischen Historiographie und Quellenkunde. 1881. 145.

$\left.{ }^{3}\right)$ Siehe Bursians Jahresberichte, 46. Band, 100.
" $"$
60.
64.

Beecke, Aristidesquellen. 
Quibus fontibus Aelius Aristides in componenda declama-

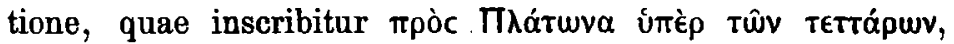
usus sit, diss. Greifswald 1884. Dabei hat Letzterer, „cum eae res quae in hac oratione (úr. $\tau$. $\tau$.) afferuntur, magna ex parte otiam in Panathenaico inveniantur" mehrfach auch auf die Quellen des Panathenaikos eingehen müssen. An die Haasschen Untersuchungen knüpft an Haury, Quibus fontibus Aelius Aristides usus sit in declamatione, quae inscribitur TavaOnvaikóc, diss. Augsburg 1888. Zuletzt sind dann untersucht

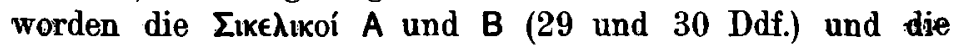
Aєยктрікоí A-E (33-37 Ddf.) durch Burchardt, Quibus ex fontibus Aristides in orationibus Siculis et Leuctricis seribendis hauserit, diss. Rostock 1895. Für eine Untersuchung über die Benutzung eines einzelnen Schriftstellers seitens des Aristides liegt vor die Arbeit von Schröder, Thucydidis historiarum memoria quae prostat apud Aristidem, Aristidis scholiastas, Hermogenem, Hermogenis scholiastas, Aristophanis

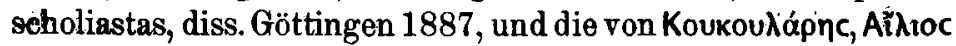

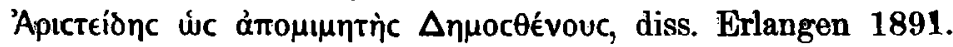

Während Bauer und Haas zu dem Resultat kamen, daB Aristides im wesentlichen aus den auch uns noch erhaltenen klassischen ${ }^{1}$ ) Historikern (Herodot, Thukydides, Xenophon, Plutarch), Rednern (besonders Demosthenes, Isokrates) Philosophen (Platon, Aeschines ${ }^{2}$ ) und Dichtern (Homer, Simonides, Pindar, Tragiker und Komiker) geschöpft, auch Burchardt diese als in der Hauptsache von Aristides verwertet eruierte, glaubte Haury betreffs des Tava $\eta$ vaı́óc beweisen zu können: "Aristides in rebus gestis narrandis in Panathenaico uno usus est Ephoro", betreffs der anderen in Betracht kommenden Rede, ír. $\tau$. $\tau$. ,in declamatione, quae inscribitur únè $\tau \hat{\omega} v$ $\tau \in \tau$ Tápwv, fere uno". (a. a. 0. 32.)

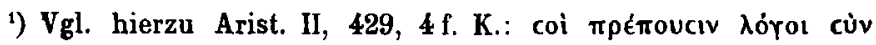

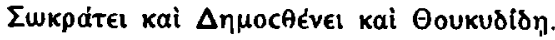

2) Vgl. Hirzel, Der Dialog I, 130, 2. 3. 4. 132, 1. 133, 1. 5. 139, 1. 140, 2; ferner Keil, Die solonische Verfassung in Aristoteles Verfassungsgeschichte Athens. 1892, 218, 2 und Arist. II, 97, 5. K. 
Wäre dieses Resultat richtig ${ }^{1}$ ), so müßte der Pan. ${ }^{8}$ ) für uns eine besondere Wichtigkeit beanspruchen, indem uns in diesem Falle in ihm eine sonst verloren gegangene Überlieferung, die des Ephoros, in neuer Brechung neben Diodor vorläge, wir also diese Rede als selbständige Quelle neben den anderen Schriftstellern zu betrachten und zu verwerten hätten. Bei der Wichtigkeit der aus dieser Ansicht sich eventuell ergebenden Konsequenzen wird die im folgenden gegebene Nachprüfung des von Haury gewonnenen Resultates als durchaus berechtigt erscheinen. Wenn sich meine Ausführungen im wesentlichen gegen Haury richten, so ließ sich dabei doch eine durchgehende Polemik gegen diesen vermeiden; es genügt ja, in erneuter Nachprüfung die Quellen darzulegen; daraus wird sich Haurys Auffassung im einzelnen Fall von selbst korrigieren.

Im Laufe der Untersuchang stellte sich heraus, daß die Verbältnisse für die Quellenforschung bei Quatt.2) und den $\mu \in \lambda \dot{t} \tau a l$ wesentlich verschiedene sind von denen unserer Rede. Lassen sich nämlich bei jenen Reden anhaltend größere zusammenhängende Partieen auf einen und denselben Autor zurückführen, so ist im Pan. das historische Material derart aus den verschiedensten Quellen zusammengeschweißt und sind die Materien, die aus den verschiedenen Autoren geflossen sind, in einer solchen Weise durcheinander gewürfelt, daß es nicht möglich war, die Quellenanalyse in der Gestalt zu geben, wie dies Haas z. B. tat, nämlich von Anfang an unter die einzelnen Schriftsteller den aus ihnen stammenden Stoff zu rubrizieren. Der Grund dafür liegt in der verschiedenen Arbeitsweise; während Arist. in den übrigen Reden strecken-

1) Mit Recht bestritten von Keil, Wochenschrift für klass. Philologie, 1891 ( $\mathrm{Nr}$, 45), 1225-1228; s. dagegen Bursians Jahresberichte, Band 62, 81 f, wo aber in einem Atem zugestanden wird: „Aber Ephoros dürfte schwerlich eine ausschließliche Quelle oder auch nur Hauptquelle des Rhetors, der doch nach seinen Schriften ein vielbelesener Mann war, gewesen sein".

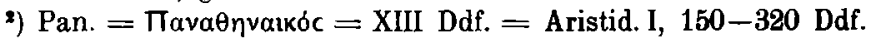

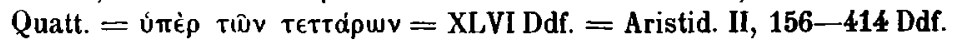


weise bestimmten Autoren folgt, hat er im Pan. die historischen Angaben teils, wie sich zeigen wird, aus dem Gedächtnis heraus niedergeschrieben, teils entsprechend der gewählten Argumentation auseinander gerissen und gemodelt. So mußten denn hier die in Betracht kommenden Ausführungen partieenweise analysiert werden, soda $B$ auf diese Weise das in vielen. Fällen so überaus kunstvoll gesponnene Gewebe der Rede Stelle für Stelle aufgelöst wird, nach der Art also, wie dies Haury, wenigstens für den größeren Teil der Rede, und ebenso Burchardt in seiner Arbeit versucht haben. Wir haben dabei die historisch-chronologische Reihenfolge der Ereignisse unserer Analyse zugrunde gelegt und nicht in der Reihenfolge die historischen Angaben behandelt, in der wir sie bei Arist. lesen; denn dieser hat die Taten Athens lediglich wie er sie jedesmal und wann er sie jedesmal brauchte, in seine jeweilige Argumentation eingereiht. Es läßt sich daher bei ihm, abgesehen von der im allgemeinen ja ziemlich genau durchgeführten Scheidung zwischen Ereignissen der Mndıká, der

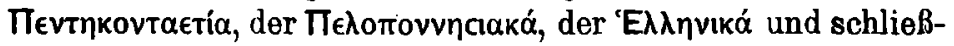
lich der $\Phi_{1} \lambda_{1} \pi \pi ı \alpha \alpha$ von einer Reihenfolge der Tatsachen im einzelnen, d. h. innerhalb dieser größeren Partieen überhaupt kaum reden. Hierher gehören auch die so außerordentlich häufigen, aus demselben Grunde natürlich notwendig gewordenen, Wiederholungen ${ }^{1}$ ) von bereits Gesagtem, auf die wir gerade in dieser Rede stoßen; und in nicht wenigen Fällen ist es nur durch eine genaue Zusammenstellung eben dieser für die einzelnen Angaben möglich gewesen, in jedem einzelnen Falle den Umfang des von Arist. tatsächlich $\mathrm{Ge}-$ wuBten festzustellen und danach diese oder jene Quelle als benutzt zu bezeichnen. War es Haas oder Haury bisweilen unmöglich, überhaupt eine Quelle zu eruieren, oder sind sie zu unhaltbaren Resultaten gelangt, so ist dies des öfteren nur dem Unstande zuzuschreiben, daß sie nach solchen scheinbaren Wiederholungen nicht suchten, weil sie nicht bedachten,

1) Betreffs derer vgl. z. B. schol. Aristid. III, 100, $19 \mathrm{ff} .171,7 \mathrm{ff}$. Ddf. auch J. Reiske, animadv. ad Graecos auct. 1761. vol. III, XIX. 
daß Arist. in dieser Rede entsprechend der jeweiligen Argumentation bereits Gesagtes in oft durchaus anderem Gowande, anderer Schattierung noch einmal bringt, und eben wegen der Ummodelung im einzelnen, ohne sich direkt zu wiederholen, auch wieder bringen konnte.

Die Anlage unserer Arbeit wird folgende sein :

Den ersten und Hauptteil derselben wird die Analyse der in Betracht kommenden Stellen der Rede einnehmen.

Im zweiten Teile werden die Ergebnisse in einer dreifachen Weise zusammenzufassen sein.

Einmal ist, $n$ m Aristides' Arbeitsweise noch klarer erkennen zu lassen und gleichzeitig überhaupt eine Übersicht des von ihm gebotenen historischen Materials zu geben, lediglich eine Zusammenstellung der verherrlichten Taten mit Quellenangabe vonnöten; dann eine Rubrizierung derselben nach den als Quellen in Betracht kommenden sicher bestimmbaren Einzelantoren, um nach dieser Seite ein Bild zu geben von dem Umf́nge des von Aristides gekannten und benutzten Quellenmaterials. Damit wird zu gleicher Zeit das Bild der literarischen Studien der Rhetoren jener Zeit schärfere Umrisse erhalten.

Einem dritten und letzten Abschnitte dieses zweiten Teiles endlich ist vorbehalten die Summe alles dessen, was wir einer bestimmten Quelle nicht zuweisen können; hierunter fällt zunächst

a) das was wir wohl einer Quelle überhaupt, aber keiner bestimmten zuzuweisen vermögen, und zwar

a) was mit diodoreischer Überlieferung in Übereinstimmung befunden wurde;

b) was wir an Angaben finden, die mit mehreren Autoren so übereinstimmen, daß eine Entscheidung darüber, w $\theta$ r von diesen in jedem einzelnen Fall als ev. Quelle in Betracht kommt, nicht zu geben ist, d. h. also Angaben, welche die rhetorische Vulgattradition repräsentieren; endlich

B) was wir überhaupt keiner Quelle zuweisen können, singuläre Angaben.

Für das Verständnis der folgenden Einzeluntersuchung 
scheint es angezeigt, die Disposition der Rede in kurzer Schematisierung vorzuführen. So wird man leichter überschauen, in welcher Verbindung und unter welchen Gesichtspunkten die einzelnen Nachrichten teilweise oder ganz, einmal oder wiederholt, auftreten:

\section{Disposition der Rede.}

A. Прооі $\mu\left(\alpha^{1}\right):$ I, 150,1-154, 20.

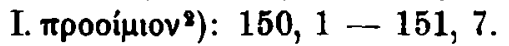

II. $\pi$ pooi tov $\left.^{3}\right): 151,7-154,5$.

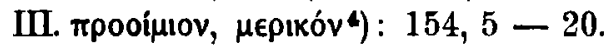

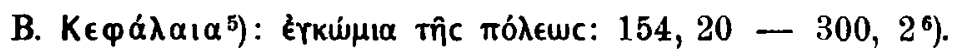

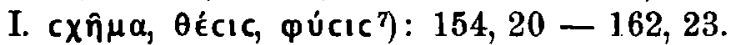

a: $154,20-162,5$.

$b^{8)}: 162,6-162,23$.

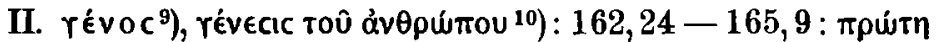

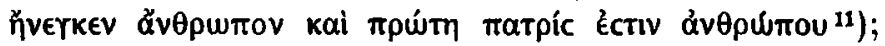

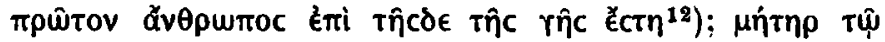

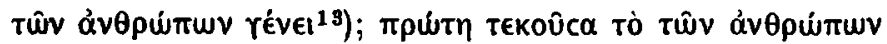
révoc ${ }^{14}$ ).

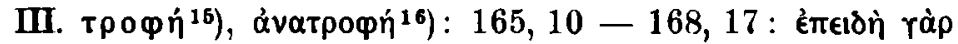

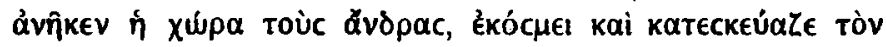

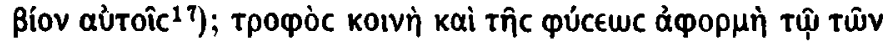

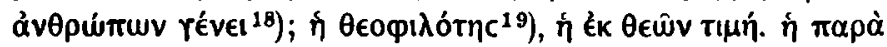

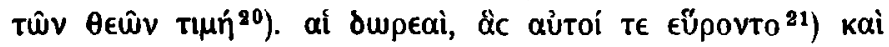

318, 10.

2) III, 1, 1. 1, 25. 2, 12. $3,13$.

s) III, 6, 16.

4) III, 15, 15.

s) III, 15,15 .

6) s. u. S. 9, 6 .

7) I, 156,12. 159,18.

160, 1. 161, 11. 13. 20.

III, 17, 18, 18-20. III, $18,3-4.19,10$ f. $26,30$. 31,12 ff. 33,30 f. 34 ,
11-13.318, 7-12, vgl.

Menand., Rhet. gr. III,

$344,15 \mathrm{ff} .346,29 \mathrm{ff}$. Sp.

$=$ Der Rhetor Menan-

der... von C. Bursian, $44 \mathrm{ff}$.

o) I, 162,6 .

9) I, 172, 11. III,

$68,27$.
10) III, 48, 17.
11) I, 163, $6 \mathrm{ff}$
18) $1,166,5$.
19) I, 163, $9 \mathrm{f}$.

14) I, 300, 9

15) I, 172, 11 III, 48 , 17. $318,13$.

16) III, 57, 7 ff. 68,

$27 \mathrm{f}$.

17) I, $165,12$.

19) I, 163, 10.

19) vgl. Rhet. gr. III, $361,22+30$ ff. 362,1 ff. Sp. u. Arist. I, 166, 9 f.

so) I, 166, 14. 168, 9.

$301,6$.

11) I, 172, 12 . 


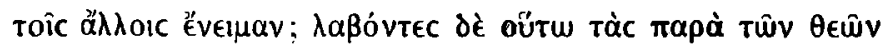

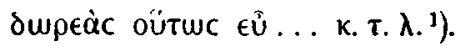

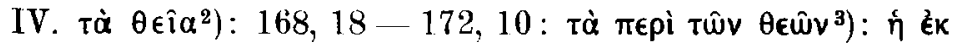

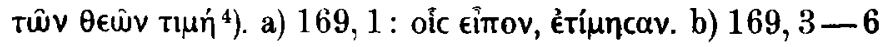

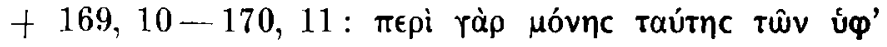

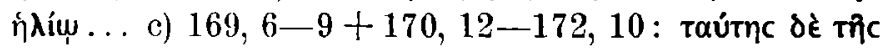

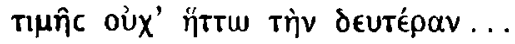

V. í $\varphi\left(\lambda \alpha v \theta \rho \omega \pi \alpha^{5}\right): 172,11-186,3$ : eủeprecía $\left.{ }^{6}\right)$. ठ̌cn

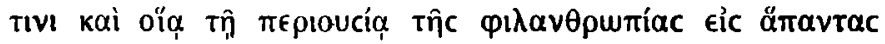

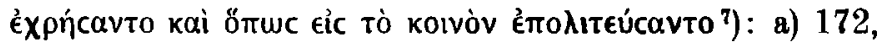

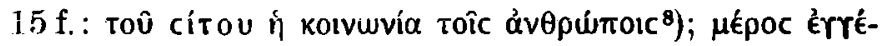

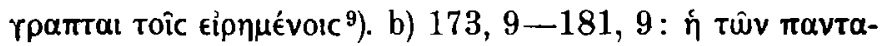

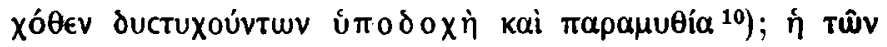

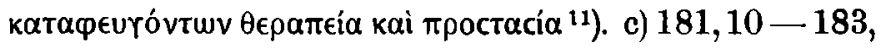

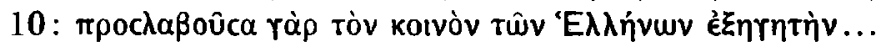

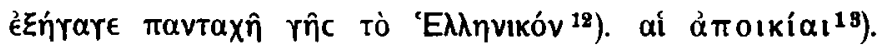

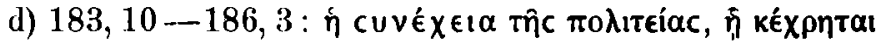

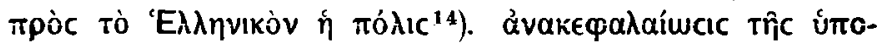

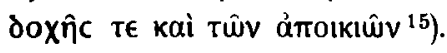

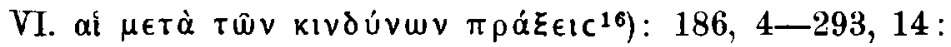

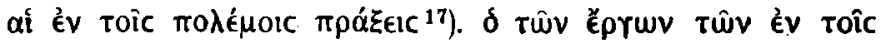

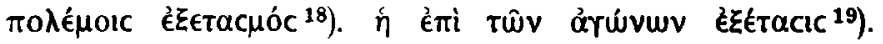

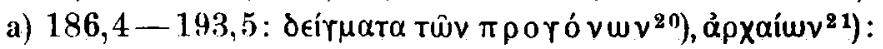

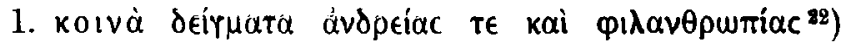

\begin{tabular}{|c|c|c|}
\hline ) $\mathrm{I}, 166,15$. & 9) ], 172,15 f. III, 68, & 33. 3 \\
\hline 2) I, 168, 18. 301, 万ff. & $26-69, \quad 15.86,21$ & 6. 138,3 . \\
\hline III, $57,15.58,3$. & 136,5 & 14) I, 173, 3. 183, 11 . \\
\hline 3) III, 56, 32 . & $\left.{ }^{10}\right) 1,173,9$ & $184,18.24 \mathrm{f}$. \\
\hline$\left.{ }^{4}\right)$ I, 169, 1. 6. 172. & 11) I, 186, 22. 300,14. & 15) III, 93, 1 . \\
\hline $\begin{array}{l}11 . \\
\text { 5) I, } 173,1.186,14 .\end{array}$ & $\begin{array}{l}\text { vgl. auch III, } 68,33 \mathrm{ff} \text {. } \\
69,4 \text { f. } 69,25,81,24 .\end{array}$ & $\begin{array}{l}\text { 16) I, 186, 5. } \\
17) \text { I, 300, } 13 .\end{array}$ \\
\hline 187, 2. III, 57, 15. 68, & 86,1 ь. 18. $87,28.94$, & 18) I, 186, 16 \\
\hline 28. $69,5.69,24.100,4$ & $\begin{array}{l}35.95, \\
138.1\end{array}$ & $\begin{array}{l}\text { 19) I, } 187,3 \text {. } \\
20) \text { I } 193.7\end{array}$ \\
\hline III, 81, 23. & 12) I, 181, 13 . & 2) $\mathrm{I}, \mathbf{1 8 9}, \mathbf{1 0}$ \\
\hline 7) I, 173, 1 ff. vgl. I, & 13) $1,182,22.186,18$ & 22) I, $186,23 \mathrm{f} .18$ \\
\hline $\begin{array}{l}\text { 183, 11. } 184,24 \text { ff. } 185, \\
1 \text { ff. III, } 96,9 .\end{array}$ & $\begin{array}{l}300,15 \text {. III, } 68,35.69 \\
25 \text { f. } 81,24.86,19.87\end{array}$ & $\begin{array}{l}\text { 15. } 188,3.9 .11 .18 . \\
189,8 \mathrm{f} .\end{array}$ \\
\hline ) 1 & 12.2 & \\
\hline
\end{tabular}




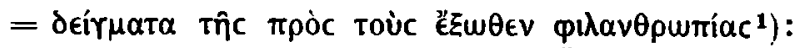
187, 1 - 189, 8. (186, 4-187, 1 Übergang).

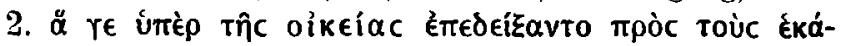

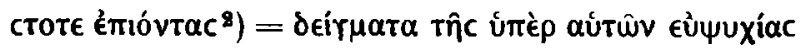

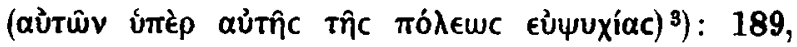
$10-191,4$.
\end{abstract}

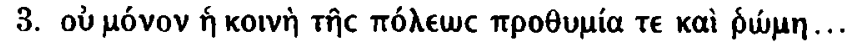

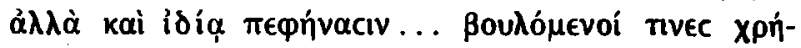

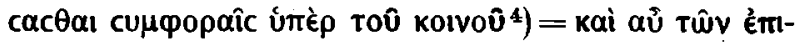
甲avî̀ 5): $191,4-193,5$.

b) $193,6-292,10$ : oí véoเ $\pi$ ó $\lambda \in \mu 0$ I $^{6}$ ).

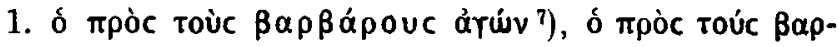

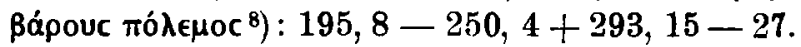
(193, 6 - 195, 8 Übergang).

a) ó Ėrì tìc oỉkeíac ${ }^{9}$ ): $195,8-242,16$.

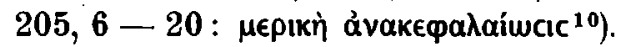

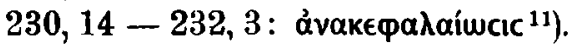

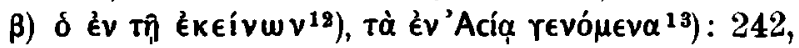
$16-250,4$.

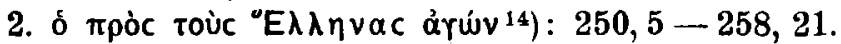

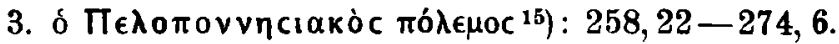
$265,14-274,7$ : cúrkpicic $\left.{ }^{16}\right)$.

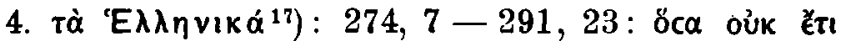

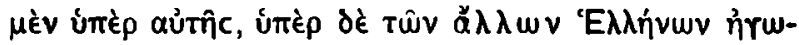

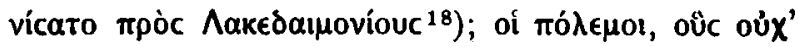

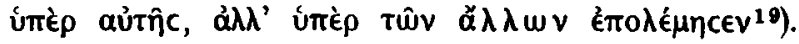

276, $13-281,15$ : cúrkpıcıc $^{20}$ ).

5. $\left.T \grave{\alpha} \Phi_{1} \lambda_{\imath} \pi \pi \imath k \grave{\alpha}^{21}\right): 291,23-292,10$.

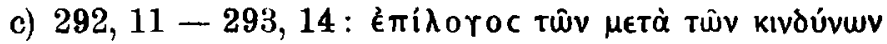

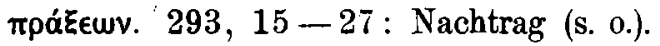

1) I, 193,7 .

2) $1,189,10$.

3) $\mathrm{I}, 193,8$.

) I, 191, 6 .

b) I, 193,9 .

8) III, 115, 5 .

7) I, $258,3$.

๑) I, 250, 5 .
9) I, 250, 6 .

10) III, $137,18 \mathrm{ff}$.

11) III, 183, 35 ff.

12) I, 250, $6 \mathrm{f}$.

1s) III, 203, 8 .

14) I, 258, 4 .

15) III, 209, 34. 228,

20. 26. 229,4 .
18) III, 242,11 f. 258 , 20 f. 259,14 .

17) III, 259,16.260,1.

18) III, $259,34 \mathrm{f}$.

19) III, 260,9 ff.

20) III, 265, 14. 276 ,

16.

21) III, 295, 34f.296, 3. 


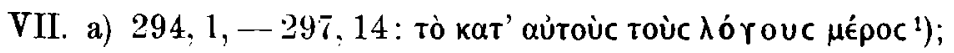

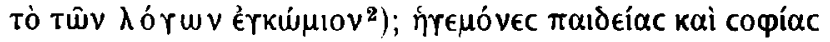
$\left.\dot{\alpha} \pi \alpha \dot{c} c \eta c^{3}\right)$.

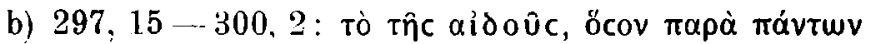

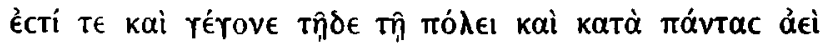

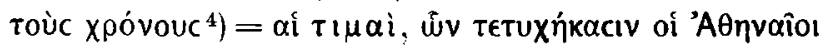

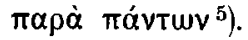

C. 'Emiגorol $\left.{ }^{6}\right): 300,2-320,10$.

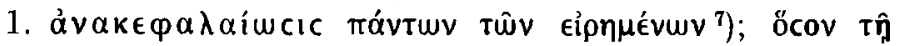

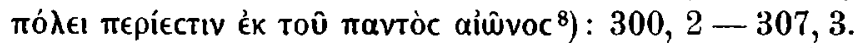

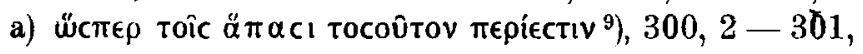

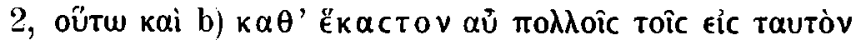
pépouciv árád $\left.\lambda \lambda \in \tau \alpha{ }^{9}\right): 301,3-307,3$.

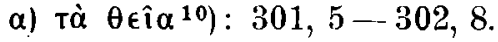

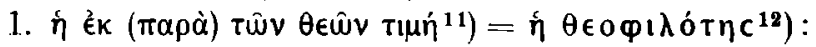
$301,7-302,2$.

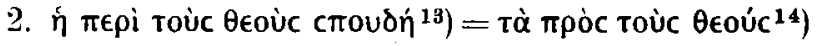

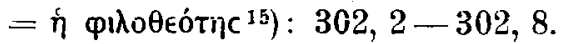

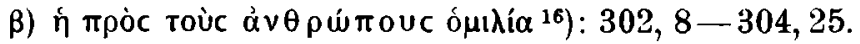

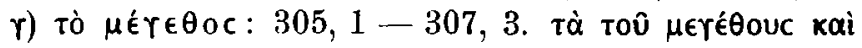

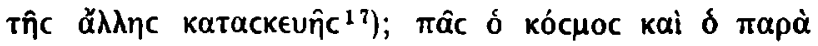

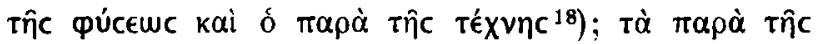

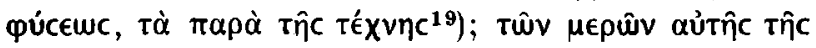

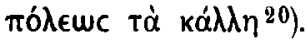

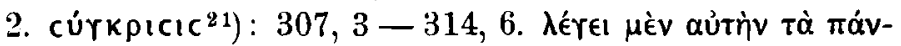
TwV Ěx

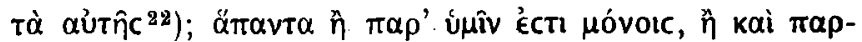

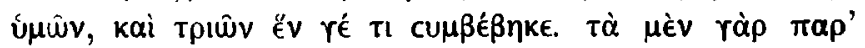

1) $\mathrm{I}, 153,10 \mathrm{ff} .294,5$.

I, 318, 5-16.

2) III, 300, '9.

$3 \mathrm{I}, 296,21$.

4) I, 297, $15 \mathrm{ff}$.

5) vgl. Rhet. gi. III, 365,10 ff. Sp. Arist. I, 298, 6. 20. III, 307, $27 \mathrm{ff}$.

\%) III, 33, 32. 58, 2 . 307, 27. Die Alten

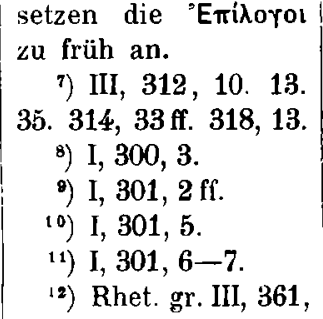

21. $30 \mathrm{ff}$.

1s) I, 301, 6 .
14) I, 302, 2.

1s) Rhet. gr. Sp. III, 361, 22. 24. 362, 22.

16) I, $302,8 \mathrm{ff}$.

17) I, 305, 1.

18) I, 305, 10 .

19) I, 305, 22. 306, 1.

$\left.{ }^{20}\right)$ III, 318, 14f. vgl.

III, 33,32 ff. 58,1 ff.

21) III, 318, 13.321, 21.

2) III, 321, $22 \mathrm{f}$. 